\title{
'Marker' grazing-incidence X-ray photon correlation spectroscopy: a new tool to peer into the interfaces of nanoconfined polymer thin films
}

\begin{abstract}
Naisheng Jiang ${ }^{1}$, Maya K Endoh ${ }^{1}$ and Tadanori Koga ${ }^{1,2,3}$
It is well known that physical and mechanical properties of polymeric materials confined at the nanoscale differ substantially from those of the bulk, known as the so-called 'nanoconfinement' effects. There is now growing evidence that the air/polymer interface and polymer/substrate interface have crucial roles in these nanoconfinement effects. Although most previous work has revealed deviations in the 'average' properties of entire nanometer films from the bulk, it is expected that the local properties within nanoconfined polymer thin films are different from the average ones, depending on the interplay between these interfaces. Here, we review our recent experimental results to measure local properties by utilizing the newly developed 'marker' grazing-incidence X-ray photon correlation spectroscopy (marker GIXPCS). The results provide new insight into the nanoconfinement effects on the local viscosity distributions within single nanoconfined polymer thin films at temperatures much greater than the bulk glass transition temperature.
\end{abstract}

Polymer Journal (2013) 45, 26-33; doi:10.1038/pj.2012.184; published online 24 October 2012

Keywords: adsorbed layer; marker GIXPCS; nanoconfinement; surface reduced viscosity layer; viscosity

\section{INTRODUCTION}

The effects of confinement at the nanometer scale ('nanoconfinement effects') on polymeric materials have been extensively investigated over the past two decades. As the early elegant studies on the glass transition behavior of nanoconfined polymer thin films by Keddie, Jones and Cory, ${ }^{1,2}$ there has been growing evidence that the physical and mechanical properties of polymer chains confined at the nanoscale, including the glass transition temperature $\left(T_{\mathrm{g}}\right)$, viscoelastic properties, interdiffusion and physical aging, vary significantly from the bulk properties. As many sophisticated devices are moving toward nanometer scales, a more thorough understanding of structure-property relationships under nanoconfinement is of great importance in the development of new nanotechnologies.

To investigate nanoconfinement effects on structure and dynamics, spin-cast ultrathin polymer films (typically $<100 \mathrm{~nm}$ in thickness) prepared on solid substrates have been investigated as model systems using various experimental techniques, such as in situ spectroscopic ellipsometry, ${ }^{1-7}$ temperature-dependent X-ray photoelectron spectroscopy, ${ }^{8}$ Brillouin light scattering, ${ }^{9-11}$ atomic force microscopy, ${ }^{12-17}$ scanning force microscopy, ${ }^{18}$ positronium annihilation lifetime spectroscopy, ${ }^{19}$ fluorescence labeling/multilayer technique, ${ }^{20-22}$ dielectric relaxation spectroscopy, ${ }^{23-32} \mathrm{X}$-ray reflectivity $^{33-35}$ and X-ray photon correlation spectroscopy (XPCS). ${ }^{36-38}$
Despite many exciting experimental findings, the underlying mechanisms of nanoconfinement effects are not fully understood yet. This lack of understanding is mainly because the above experimental techniques measure 'average' properties of entire nanometer films, while the local behavior might be heterogeneous, depending on the interplay between the two interfaces, that is, the air/polymer interface and substrate/polymer interface. Therefore, it is crucial to develop an advanced nanoscale tool to determine the in situ local properties of polymeric nanomaterials as a function of the distance from the interfaces.

This review highlights our recent experimental work ${ }^{39,40}$ on the heterogeneous rheological properties within ultrathin polystyrene (PS) films associated with the interplay between the two interfaces. The technique used was 'marker' grazing-incidence XPCS (marker GIXPCS) using gold nanoparticles embedded in ultrathin PS films. The next section introduces the basic principle of marker GIXPCS: the random drifting (the Brownian motion) of the individual markers adequately tracks the local viscosity of entangled polymer chains in the regions of interest within single PS thin films. The section 'Surface reduced viscosity layer' describes the novel characteristics of an air/ polymer interface with a viscosity that is $\sim 30 \%$ lower than that of the bulk at $T \gg T_{\mathrm{g}}$, while the dynamics of the polymer chains are still governed by the reptation mechanism. The section 'Effects of

${ }^{1}$ Department of Materials Science and Engineering, Stony Brook University, Stony Brook, NY, USA; ${ }^{2}$ Chemical and Molecular Engineering Program, Stony Brook University, Stony Brook, NY, USA and ${ }^{3}$ Department of Chemistry, Stony Brook University, Stony Brook, NY, USA

Correspondence: Dr MK Endoh or Professor T Koga, Department of Materials Science and Engineering, Stony Brook University, Stony Brook, NY 11794 2275, USA. E-mails: tadanori.koga@stonybrook.edu or maya.koga@stonybrook.edu

Received 2 August 2012; revised 1 September 2012; accepted 2 September 2012; published online 24 October 2012 
adsorbed layers formed on the substrate surface' focuses on the impact of the very thin adsorbed layer (several nanometers in thickness) formed at the polymer/substrate interface, which overcomes the effect of the surface reduced viscosity layer and results in a significant increase in the local viscosity approaching the substrate interface. Finally, the section 'Conclusions' provides some concluding remarks.

\section{MARKER GIXPCS}

Photon correlation spectroscopy using laser light is a well-established tool ${ }^{41}$ for probing the dynamic properties of matter by analyzing the temporal correlations among photons scattered by the matter. During the past decade, the development of third-generation synchrotron radiation sources has made it possible to extend photon correlation spectroscopy from the optical region into the X-ray domain. The use of the brilliant X-rays enables us to probe the dynamic properties of systems on molecular length scales and to use optically dense samples that are not accessible to conventional photon correlation spectroscopy. XPCS has been extended into the sub-microsecond range of temporal resolution, and the advent of an X-ray free-electron laser would further provide a unique opportunity to identify chemical and biological phenomena with unprecedented temporal resolution (femtosecond regime). Thus, XPCS has great potential to impact the study of a variety of nonequilibrium phenomena. Because this article focuses on GIXPCS, the reader is referred to some recent reviews ${ }^{42-44}$ for discussion of transmission XPCS, including the fundamentals and other promising fields of application.

Recently, Sinha and co-workers have demonstrated that the GIXPCS technique is the most direct and unambiguous way to study the lateral dynamics of polymer thin films prepared on solid substrates based on the surface capillary wave fluctuations. ${ }^{36,37,45-49}$ Using normal hydrodynamic theory for capillary wave fluctuations on viscous liquid films, they have shown that the viscosity of single PS films that are several tens of nanometers thick is similar to that of the bulk. ${ }^{36}$ However, it should be emphasized that the viscosity obtained by this methodology corresponds to the 'average' one over the entire thickness ${ }^{36}$ because the capillary fluctuations propagate into the film interior. Thus, another novel strategy is required to determine the local rheological properties of polymer thin films as a function of the distance from the interfaces and to clarify to what extent the two interfacial effects propagate into the film interior. It was this question, which has not yet been answered theoretically or experimentally, that motivated our present study.

There is an earlier transmission XPCS result ${ }^{50}$ showing that the viscosity of glycerol solvent can be reasonably determined from the Brownian motion of gold particles embedded in the liquid as markers. This type of strategy is the so-called passive microrheology approach or tracer diffusion approach, which takes advantage of the inherently strong X-ray contrasts of the markers. To apply this strategy to a given system, the concentration of the markers should be sufficiently low enough, and the markers should be neutral to the surrounding materials; otherwise, the effects of particle-fluid interactions and the interference among the markers would make the XPCS analysis complicated. If the motions of the isolated markers obey Gaussian statistics, the mean squared displacement of the markers in the given time domain, $\left\langle\Delta r^{2}(t)\right\rangle$, can be extracted from the following relationship: ${ }^{42}$

$$
g_{2}(q, t)=1+A \exp \left[-\left\langle\Delta r^{2}(t)\right\rangle q^{2} / 3\right]
$$

where $g_{2}(q, t)$ is the normalized intensity-intensity time autocorrelation function of the given wave vector transfer $(q)$ at time $(t)$, and $A$ is a speckle contrast $(0<A<1)$. In the case of the GIXPCS measurements, $g_{2}\left(q_{/ /}, t\right)$ can be obtained from the following equation:

$$
g_{2}(q / /, t)=\left\langle I\left(q / /, t^{\prime}\right) I\left(q_{/ /}, t^{\prime}+t\right)\right\rangle /\left\langle I\left(q / /, t^{\prime}\right)\right\rangle^{2},
$$

where $I\left(q_{/ /}, t^{\prime}\right)$ refers to the scattering intensity of the in-plane wave vector transfer $\left(q_{/ /}\right)$at time $(t)^{\prime}$. The brackets $\langle>$ refer to averages over time $t^{\prime} . g_{2}\left(q_{/ /}, t\right)$ is related to the normalized intermediate structure factor, $\left[f\left(q_{/ /}, t\right)\right]$, via $g_{2}\left(q_{/ /}, t\right)=1+A\left[f\left(q_{/ / /}, t\right)\right]^{2}$. For purely diffusive particle motions, the mean squared displacement of the markers over $t$ follows the relation, $\left\langle\Delta r^{2}(t)\right\rangle=6 D t$, where $D$ is the diffusion constant of the markers. The normalized intensity-intensity time autocorrelation function is then rewritten as follows:

$$
g_{2}(q / /, t)=1+A \exp \left[-2 D q_{/ /}^{2} t\right]
$$

The viscosity $(\eta)$ of the surrounding material is inversely proportional to $D$ via the well-known Stokes-Einstein relation:

$$
D=\frac{k_{B} T}{6 \pi \eta R},
$$

where $R$ is the radius of the markers, $k_{\mathrm{B}}$ is the Boltzmann constant and $T$ is the absolute temperature. Here, we adopted this concept for GIXPCS, which is denoted as 'marker GIXPCS' hereafter.

The schematic view of the marker GIXPCS setup is shown in Figure 1. The marker GIXPCS experiments were performed at the 8-ID beam line at the Advanced Photon Source in Argonne National Laboratory with an X-ray energy of $7.5 \mathrm{keV}$. Six different molecular weights $\left(M_{\mathrm{w}}\right)$ of PS $\left(M_{\mathrm{w}}=30,65,123,200,290\right.$, and $\left.400 \mathrm{kDa}\right)$ having narrow polydispersities $\left(M_{\mathrm{w}} / M_{\mathrm{n}}=1.02-1.05\right)$ were used. Thiol-functionalized (octadecanethiol $\left.\left(\mathrm{C}_{18} \mathrm{H}_{37} \mathrm{SH}\right)\right)$ Au nanoparticles were prepared as markers using the one-phase synthesis method. ${ }^{51}$ The average radius of the core Au particles was $1.5 \pm 0.2 \mathrm{~nm}$, and the thickness of the $\mathrm{C}_{18} \mathrm{H}_{37} \mathrm{SH}$ shell layer was $\sim 1.3 \mathrm{~nm}$. Solutions of PS were first prepared in toluene. The Au nanoparticles (the volume fraction was $0.2 \%$ ) were then added to the PS solutions, which were further sonicated for $30 \mathrm{~min}$. The PS/Au thin films were prepared by spin-casting onto HF-etched Si substrates and annealed for up to 4 days in a vacuum of $10^{-3}$ Torr at $T=170{ }^{\circ} \mathrm{C}$, which is significantly higher than the $T_{\mathrm{g}}$ of PS. Four different film thicknesses $(32 \mathrm{~nm}$, $57 \mathrm{~nm}, 128 \mathrm{~nm}$ and $235 \mathrm{~nm}$ ) were prepared. We confirmed that the Au particles were well dispersed in all of the films and no surface segregation of the Au nanoparticles occurred at the two interfaces before or after the marker GIXPCS experiments. The in-plane

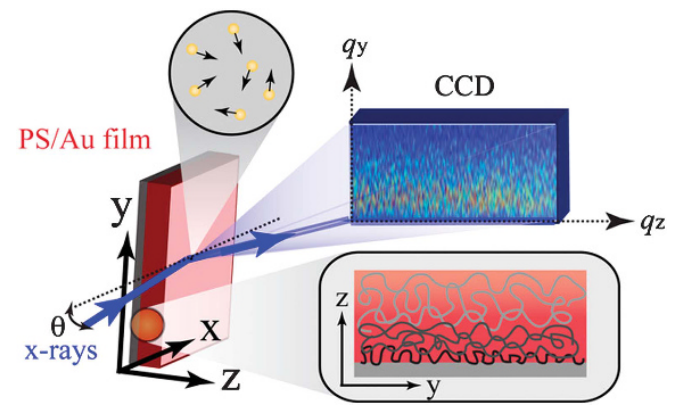

Figure 1 Schematic diagram of the marker GIXPCS experiments. The use of resonance enhanced X-ray scattering for marker GIXPCS enables the probing electrical field to be intensified by more than one order of magnitude in the regions of interest within single polymer films. 
dynamics of the Au nanoparticles were monitored by recording time series of speckled scattering intensity using a CCD camera.

In addition, two illuminating modes with different incident angles $(\theta)$ were used for the GIXPCS measurements to explore the marker dynamics at the topmost surface ('surface-mode') and at the nearcenter region of the film ('center-mode') independently. ${ }^{39,40}$ In the surface-mode, an incident angle of $\theta=0.15^{\circ}$, which is just below the critical angle of the total external reflection of the PS films with the X-ray energy $\left(\theta_{\mathrm{c}}=0.16^{\circ}\right)$, was used. According to previous calculations of the electric field intensity based on Parratt's exact recursive method, ${ }^{52}$ the electric field intensity in the surface-mode is 3-4 times higher than that of the incident beam within the topmost surface region ( $\sim 9 \mathrm{~nm}$ in thickness), and it then decays exponentially into the film interior. Hence, the scattering intensity in the surfacemode is dominated by a surface region of thickness of $\sim 9 \mathrm{~nm}$. For the center-mode, we utilized the 'first resonance mode' $\left(\theta>\theta_{\mathrm{c}}\right)^{53}$ in which resonance enhancement of the electric field intensity in a polymer film takes place and resonance-enhanced X-rays are significantly intensified at a position close to the center of the film (Figure 2). Use of this mode also allows us to reduce the scattering signals from both interfacial regions drastically as shown in the inset of Figure 2.

Figure $3 \mathrm{a}$ shows representative normalized intensity-intensity time autocorrelation functions, $g_{2}$, for the Au nanoparticles embedded in the PS film $\left(M_{\mathrm{w}}=123 \mathrm{kDa}, 128 \mathrm{~nm}\right.$ thick $)$ in the surface-mode. All GIXPCS measurements were performed at high temperatures $\left(156^{\circ} \mathrm{C} \leqslant T \leqslant 186^{\circ} \mathrm{C}\right)$ under vacuum to avoid the so-called 'hyperdiffusive dynamic behavior ${ }^{\prime 54,55}$ observed near $T_{\mathrm{g}}$. The $g_{2}$ functions at the five different $q_{/ /}$values were fitted by a single exponential decaying function, $g_{2}(q / /, t)=1+A \exp [-2(t / \tau)]$, where $\tau$ is the characteristic relaxation time of the Au nanoparticles. From the figure, we can see that the single exponential decaying function fits the data for the 128nm-thick film well. The same was true for the 235-nm-thick film (data not shown). On the other hand, stretched exponential decaying functions, that is, $g_{2}(q / /, t)=1+A \exp [-2(q / t)]^{\alpha}$, where $\alpha$ is the stretching exponent that characterizes the shape and ranges from

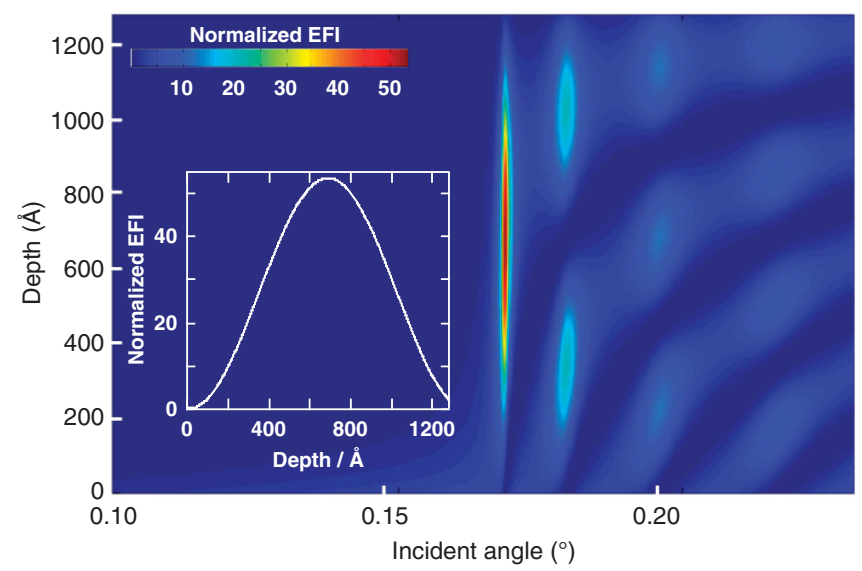

Figure 2 Calculated electric field intensity as functions of the depth from the air/polymer interface and the incident angle of the X-rays. The electric field intensity distribution in the PS/Au film was generated by the interference of $7.5 \mathrm{keV}$ X-ray plane waves reflected and refracted at the two interfaces in the film in air. The inset shows the normalized resonance enhanced X-ray electric field intensityprofile at the first resonance mode, which is 53.5 times the electric field intensity of the incident beam. Figure reproduced from Koga et al. ${ }^{39}$ with permission from the American Physical Society.
0.4 to 0.8 , were required to fit the $g_{2}\left(q_{/ /}, t\right)$ functions for the $32-\mathrm{nm}$ and 57-nm-thick films. This difference indicates the dynamical heterogeneity in the thinner films, leading to a wider spectrum of relaxation times. As shown in Figure $3 \mathrm{~b}$, the obtained $\tau$ values exhibit the power-law behavior of $\tau=1 /\left(2 D q_{/ /}^{2}\right)$ for all of the films in both modes, except for the 32-nm-thick film in the center-mode. In this case, the relaxation times $(\sim 5000 \mathrm{~s})$ were very close to the resolution of the measurements, and therefore, quantitative analysis might not be appropriate. Table 1 summarizes the $D_{\text {sur }}$ (surface-mode) and $D_{\text {cen }}$ (center-mode) values for the different thicknesses $(h)$ of the PS $\left(M_{\mathrm{w}}=123 \mathrm{kDa}\right)$ films based on the fitting of the power-law relation to the experimental data. Through a series of comparisons, ${ }^{39,40}$ we found that the marker dynamics correlate reasonably well with the viscosity of the polymer matrix via the known Stokes-Einstein relation (Equation (4)). In other words, this result verifies that the Au nanoparticles are well dispersed in the PS matrix and do not have specific interactions with the matrix. Based on the marker GIXPCS results, we discuss the inherent characteristics of the air/polymer
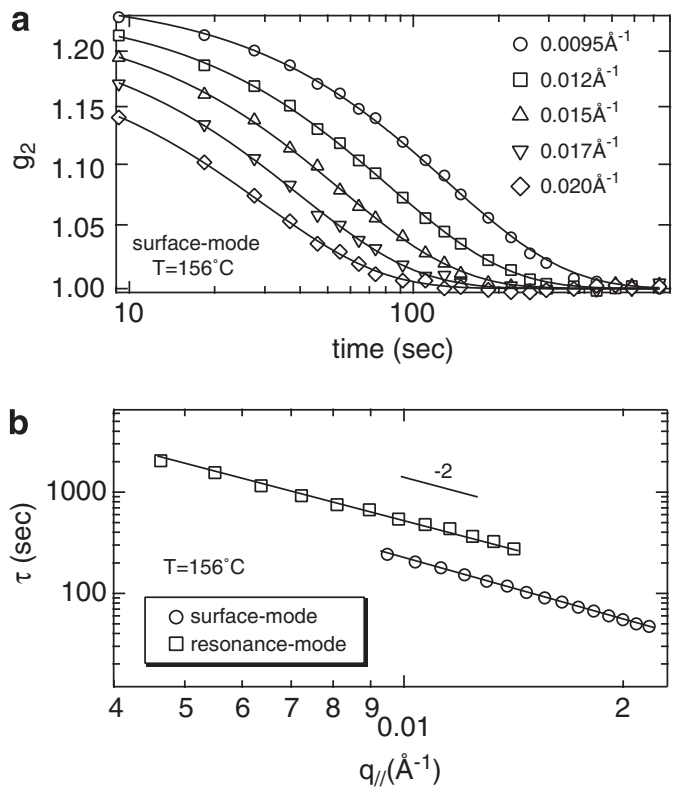

Figure 3 (a) Measured $g_{2}$ at $T=156^{\circ} \mathrm{C}$ for the PS $\left(M_{\mathrm{w}}=123 \mathrm{kDa}\right) / \mathrm{Au}$ film in the surface-mode. The solid lines are the best single exponential fits described in the text. (b) $\log -\log$ plot of $\tau$ vs $q_{/ /}$at $T=156{ }^{\circ} \mathrm{C}$ in the surface-mode and resonance mode. The solid lines correspond to the best fits of the relationship, $\tau=1 / 2 D q^{2}$, to the data. Figure reproduced from Koga et al. ${ }^{39}$ with permission from the American Physical Society.

Table 1 Measured diffusion coefficients of the Au nanoparticles and calculated viscosities of PS $\left(M_{w}=123 \mathrm{kDa}\right)$ based on the SE law at the surface and the near-center of the films at $186^{\circ} \mathrm{C}$

\begin{tabular}{lcccc}
$h(n m)$ & $\mathrm{D}_{\text {sur }}\left(n m^{2} s^{-1}\right)$ & $\mathrm{D}_{\text {cen }}\left(n m^{2} s^{-1}\right)$ & $\eta_{\text {sur }}\left(\mathrm{Nsm}^{-2}\right)$ & $\eta_{\text {cen }}\left(\mathrm{Ns} \mathrm{m}^{-2}\right)$ \\
\hline 32 & 0.016 & $-^{\mathrm{a}}$ & $7.7 \times 10^{6}$ & - $^{\mathrm{a}}$ \\
57 & 6.58 & 0.76 & $1.8 \times 10^{4}$ & $1.6 \times 10^{5}$ \\
128 & 7.80 & 5.30 & $1.5 \times 10^{4}$ & $2.2 \times 10^{4}$ \\
235 & 7.84 & 5.42 & $1.5 \times 10^{4}$ & $2.2 \times 10^{4}$ \\
\hline
\end{tabular}

aUltraslow dynamics (the very weak $q_{/ /}$dependence of $\tau$ ).

Table reproduced from Koga et al..$^{40}$ with permission from American Physical Society. 
interface and polymer/substrate interface and their impacts on the local viscosity distributions within nanoconfined single PS thin films in the subsequent sections.

\section{SURFACE REDUCED VISCOSITY LAYER}

Table 1 also shows the local viscosities in the surface-mode $\left(\eta_{\text {sur }}\right)$ and the center-mode $\left(\eta_{\text {cen }}\right)$ calculated from the $D$ values using Equation (4). It is clear that the $\eta_{\text {sur }}$ values for the $128-\mathrm{nm}$ and $235-\mathrm{nm}$ thick films are $\sim 30 \%$ lower than those at the film center, indicating the presence of a surface reduced viscosity layer at $T>T_{\mathrm{g}}$. Bodiguel and Freigny ${ }^{56,57}$ previously reported that polymers are much less viscous at the topmost surface at $T>T_{\mathrm{g}}$ as measured by the dewetting dynamics of ultrathin PS films on a liquid substrate. However, we should emphasize that the dewetting experiments provide a simple average of the local viscosity across the entire film so that the depth dependence of the local viscosity is strongly model-dependent. On the other hand, Forrest and co-workers studied the surface relaxation dynamics of glassy PS thin films using two types of experimental methodologies: the nanodeformation method ${ }^{15,16}$ and the nanoparticle embedding method. ${ }^{12-14}$ The film surface remained in a liquid-like rubbery state while the rest of the film was in the glassy state during a cooling process from the melt, proving that the viscosity is largely reduced at the free surface, that is, the air/polymer interface. However, the phenomenon occurs in a nonequilibrium glassy state. Hence, with the use of marker GIXPCS, we provide the first direct evidence of the reduced viscosity layer in the surface region of single PS melt films at $T \gg T_{\mathrm{g}}$. In addition, although the $\eta_{\text {sur }}$ value for the 57-nm-thick film is reduced to a lesser degree than those for the other films, we suppose that the same surface reduced viscosity layer still exists at the free surface. As discussed in the next section, this difference is attributed to the long-range propagation of the substrate effect. However, it is not conclusive yet whether the surface reduced viscosity layer is still present in the 32-nm-thick film due to the significant long-range perturbations.

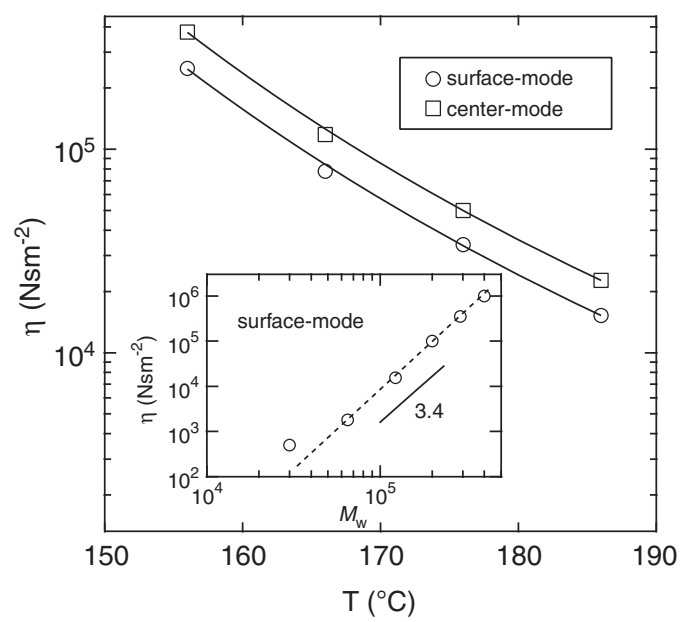

Figure 4 Temperature dependence of the viscosity of the PS $\left(M_{\mathrm{w}}=123 \mathrm{kDa}\right) / \mathrm{Au}$ film (open symbols). The solid lines correspond to the best fits of the WLF equation to the data with $c=4.0 \pm 0.5$ and $T_{\infty}=42 \pm 2^{\circ} \mathrm{C}$ for the surface-mode and $c=4.0 \pm 0.5$ and $T_{\infty}=47 \pm 2{ }^{\circ} \mathrm{C}$ for the center-mode. In the inset, a log-log plot of the viscosity of the PS/Au film as a function of the $M_{\mathrm{w}}$ of $\mathrm{PS}$ at $T=186^{\circ} \mathrm{C}$ is shown. Figure reproduced from Koga et al. ${ }^{39}$ with permission from the American Physical Society.
We further explored whether the reduced viscosity at the free surface is associated with a change in the local $T_{\mathrm{g}}$. Figure 4 shows the temperature dependence of the local viscosity for the 128-nm-thick film $\left(M_{\mathrm{w}}=123 \mathrm{kDa}\right)$. The data were fitted with the Williams-LandelFerry (WLF) equation,

$$
\log \left(\frac{\eta(T)}{\eta\left(T_{0}\right)}\right)=-c\left(\frac{T-T_{0}}{T-T_{\infty}}\right),
$$

where $T_{0}$ is an arbitrary reference temperature, $c$ is a numerical constant and $T_{\infty}$ is a fixed temperature at which $\log \left(\eta(T) / \eta\left(T_{0}\right)\right)$ becomes infinite regardless of the arbitrary choice of $T_{0}$. As shown in Figure 4, the best fits of the WLF equation to the data (solid lines) give us $c=4.0 \pm 0.5$ and $T_{\infty}=42 \pm 2{ }^{\circ} \mathrm{C}$ for $\eta_{\text {sur }}$ and $c=4.0 \pm 0.5$ and $T_{\infty}=47 \pm 2{ }^{\circ} \mathrm{C}$ for $\eta_{\text {cen }}$. Thus, the $T_{\mathrm{g}}$ values $\left(50{ }^{\circ} \mathrm{C} \text { above } T_{\infty}\right)^{58}$ at the free surface and near the center region of the 128-nm-thick film are almost bulk-like. These results are inconsistent with previous fluorescence labeling measurements using multilayer systems, which showed a large reduction in local $T_{\mathrm{g}}\left(32^{\circ} \mathrm{C}\right.$ below the bulk value $)$ at the free surface region of supported PS thin films of comparable thickness. $^{22}$ This discrepancy may imply, as several reports argued previously, ${ }^{13,56,57,59,60}$ that the viscosity reduction and the $T_{\mathrm{g}}$ reduction are two distinct phenomena because the former is related to the dynamical changes at larger scales (such as macroscopic molecular chain motions), whereas the latter involves adjustments at smaller scales (such as microscopic segmental motions or local relaxations). These macroscopic and microscopic motions might behave differently under nanoconfinement. Hence, we suppose that the reduction in the surface viscosity is inherent to the free surface of rubbery PS thin films.

The question is then what is the origin of the surface reduced viscosity? It has been proposed that the enhanced mobility at the free surface is due to either the localization of segregated chain ends ${ }^{61,62}$ or the reduction of chain entanglements on the basis of chain packing theory. ${ }^{63,64}$ To answer the question, we performed a series of marker GIXPCS experiments for PS/Au films with similar thicknesses $(\sim 130 \mathrm{~nm})$ but composed of polymers of different molecular weights. We found that when $M_{\mathrm{w}} \geqslant 123 \mathrm{kDa}$, the reduction in the local viscosity at the free surface relative to that at the film center remains almost constant as a function of molecular weight ${ }^{39}$ but gradually decreases as $M_{\mathrm{W}}$ approaches the entanglement molecular weight $\left(M_{\mathrm{c}}\right)$ of PS $\left(M_{\mathrm{c}} \sim 36 \mathrm{kDa}\right){ }^{65}$ When $M_{\mathrm{w}}<M_{\mathcal{c}}$, almost no reduction in the surface viscosity is observed, suggesting that the viscosity reduction at the free surface is related to the chain entanglements. In addition, we found that $\eta_{\text {sur }}$ follows the wellknown relationship of $\eta_{w}^{3.4}$, except in the case of the unentangled PS film $\left(M_{\mathrm{w}}=30 \mathrm{kDa}\right)$, as shown in the inset of Figure 4 . Thus, we reveal that the polymer chains at the free surface are still entangled and their motions are governed by the reptation mechanism. This finding may rule out the possibility that the polymer motion at the free surface might follow a different mode rather than a pure reptation mode. ${ }^{56,57}$ Because the depth of the probed surface region is comparable to the tube diameter $(\sim 9 \mathrm{~nm})$ of entangled PS chains, ${ }^{66}$ it is reasonable to conclude that the reduced surface viscosity is due to the reduced chain entanglements in the probed surface region. ${ }^{39}$

\section{EFFECTS OF ADSORBED LAYERS FORMED ON THE SUBSTRATE SURFACE}

Recently, Fujii et al. ${ }^{67}$ showed that PS chains are irreversibly adsorbed onto hydrogen-passivated silicon $(\mathrm{H}-\mathrm{Si})$ substrates from melts by annealing at a temperature much greater than $T_{\mathrm{g}}$ and that the 
adsorbed chains cannot be removed even after extensive rinsing with a good solvent for PS. As shown in Figure 5, they reported that the thickness of the irreversibly adsorbed layer increases with increasing annealing time until it reaches equilibrium. More importantly, they demonstrated that such adsorbed layers are formed even on very weakly attractive substrate surfaces, that is, bare $\mathrm{Si}$ substrates, although the adsorbed layer thickness is much thinner than that for the PS/H-Si systems. ${ }^{67}$ Very recently, Napolitano and Wübbenhorst ${ }^{24}$ showed that the deviation in $T_{\mathrm{g}}$ of PS thin films prepared on aluminum substrates is directly linked to the growth of the irreversibly adsorbed layer instead of the so-called 'free-surface effect'. $^{22}$ They also proposed a formation mechanism for the adsorbed layer during the long annealing process (Figure 6).

Here, we show the impact of the irreversibly adsorbed layer on the local viscosity distributions within single nanoconfined PS thin films.

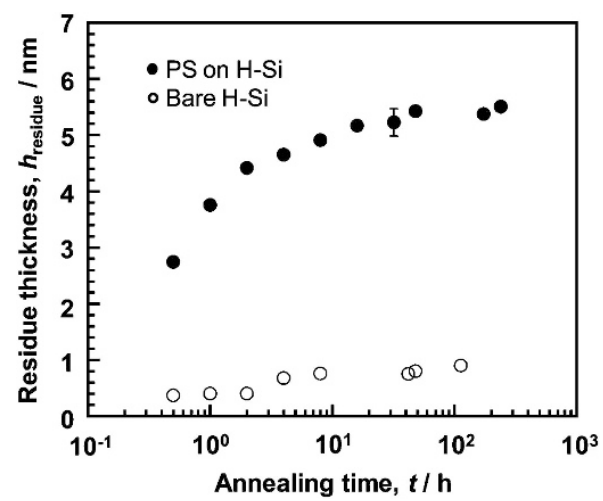

Figure 5 Annealing time dependence of the thickness of the residual film,

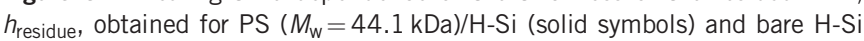
(open symbols). The annealing was performed at $T=150{ }^{\circ} \mathrm{C}$ in air. Figure reproduced from Fujii et al. ${ }^{67}$ with permission from the American Chemical Society.
To ensure the existence of the adsorbed layer, all the PS/Au films were thoroughly rinsed with toluene, a good solvent for PS, after the marker GIXPCS experiments. Using X-ray reflectivity, we found that a residual adsorbed PS layer with a thickness of $\sim 7 \mathrm{~nm}$ was formed on the H-Si substrates regardless of the original film thickness and $M_{\mathrm{w}}{ }^{40}$ We also studied the interdiffusion process for a bilayer composed of deuterated PS (dPS, $M_{\mathrm{w}}=334 \mathrm{kDa}, 51 \mathrm{~nm}$ thick) floated onto the residual adsorbed PS layer using in situ neutron reflectivity. ${ }^{40}$ No interdiffusion was observed for at least 3 days at $170{ }^{\circ} \mathrm{C}$, indicating that the dynamics of the adsorbed polymer chains are restricted even at $T \gg T_{\mathrm{g}}$. Based on these results, we can conclude that the strongly suppressed dynamics of the markers at the center of the 32-nm-thick film (Table 1) is not directly linked to this immobile adsorbed layer. Rather, the restricted dynamics are attributed to the 'reduced mobility interface layer, ${ }^{33,34,68}$ which is entangled through the network of the adsorbed layer. ${ }^{69}$ Hence, the effect of the irreversibly adsorbed layer propagates into the film interior via the reduced mobility interface layer, leading to the long-range perturbations of the local viscosity. The next question is how far this substrate effect associated with the adsorbed layer and reduced mobility interface layer can propagate into the film interior. As observed in the surface-mode for the 32-nmthick film, the substrate effect propagates to the surface region thoroughly and obscures the effect of the surface reduced viscosity layer if it exists. As a result, we observe an increase in $\eta_{\text {sur }}$ by two orders of magnitude relative to the $\eta_{\text {sur }}$ values for the other thicker films. Moreover, the surface viscosity of the 57-nmthick film is also perturbed to some extent by the substrate effect as shown by comparison with the $\eta_{\text {sur }}$ values for the 128 - and 235-nm-thick films. Hence, it is reasonable to suppose that the substrate effect propagates up to $60 \mathrm{~nm}$ from the substrate surface in the present case.

Using the temperature dependence of the viscosity according to the WLF equation (Equation (5)), the local $T_{\mathrm{g}}$ values at the surface and near the center of the $57-\mathrm{nm}$-thick film are estimated to be $\sim 100{ }^{\circ} \mathrm{C}$ and $115^{\circ} \mathrm{C}$, respectively. Thus, it is clear that the phenomenon occurs at $T \gg T_{\mathrm{g}}$ and is not related to the 'percolation transition' in a

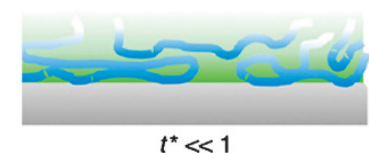

d

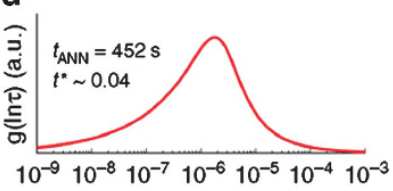

$t(\mathrm{~s})$ b

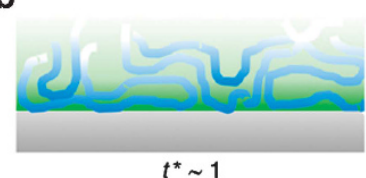

e

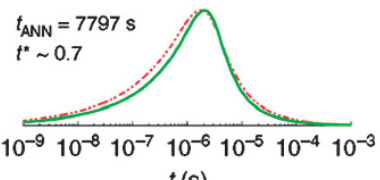

$t(\mathrm{~s})$

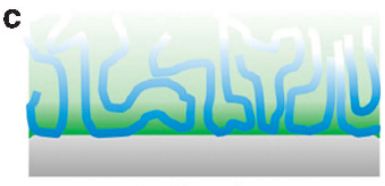

$t^{\star} \gg 1$

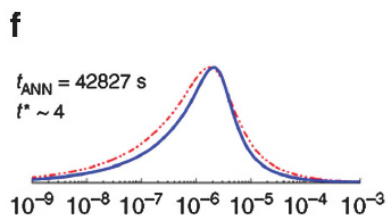

$t(\mathrm{~s})$

Figure 6 The impact of adsorption on the deviations from bulk behavior can be rationalized in terms the dimensionless parameter, $t^{*}$, corresponding to the ratio between the annealing and adsorption times. The molecular picture corresponding to the perturbation in the dynamics upon adsorption is given in $(\mathbf{a}-\mathbf{c})$. Up to $t^{*} \sim 1$, adsorption proceeds via gradual filling of the available pinning sites at the interface, which causes a decrease in the free volume between molecules and the reduction in the population of faster modes. In the latest stages of adsorption, $t^{*}>1$, the screening of previously adsorbed chains permits adsorption only upon reorganization and pinning at fewer and fewer surface sites; the corresponding lower train fraction (percentage of sequences of consecutive monomers in direct contact with the surface) yields a reduction in the slower modes. Experimental validity of this molecular picture was achieved by analyzing the distribution of the relaxation times of the dielectric spectra of a $44-\mathrm{nm}$-thick film of $\mathrm{PS}$ ( $M_{\mathrm{w}}=97 \mathrm{kDa}$ ) during annealing at $T=150^{\circ} \mathrm{C}$ at different adsorption conditions: $t^{*}=0.04(\mathbf{d}), t^{*}=0.7$ (e) and $t^{*}=4$ (f). Compared with short annealing times ( $t^{*}<<1$ (d)), adsorption induces a pronounced cutoff of the faster relaxation modes after moderate annealing times $\left(t^{*} \sim 1\right.$ (e)). Further annealing $\left(t^{*}>1\right.$ (f)) induces a reduction in the polymer segments relaxing at slower characteristic times. Figure reproduced from Napolitano and Wubbenhorst ${ }^{24}$ with permission from the Nature Publishing Group. 
supercooled polymer thin films near $T_{\mathrm{g}}{ }^{70}$ In addition, the estimated local $T_{\mathrm{g}}$ values are inconsistent with the early study by Ellison and Torkelson, ${ }^{22}$ who showed decreases in $T_{\mathrm{g}}$ at the free surface and near the center of the supported 60 -nm-thick PS film. As previously mentioned, this discrepancy may imply that the viscosityconfinement effect is not simply linked to the $T_{\mathrm{g}}$-confinement effect. However, another issue must be considered: the strength of the substrate effects. As previously reported, ${ }^{24}$ the substrate effect is strongly related to the thickness of the irreversibly adsorbed layer, which is governed by the high-temperature-annealing protocol. In addition, a recent experimental study ${ }^{25}$ showed that local $T_{\mathrm{g}}$ values at a distance of several $R_{\mathrm{g}}$ away from the attractive substrate surface depend on the annealing time. In our study, the samples were annealed at $T=170^{\circ} \mathrm{C}$, which is much $>T_{\mathrm{g}}$ of PS, for an extremely long time before the marker GIXPCS measurements, allowing the adsorbed layer to equilibrate at the polymer/substrate interface. ${ }^{40}$ However, in the case of fluorescence labeling/multilayer measurements, ${ }^{20,22}$ multilayer films should be dried under vacuum at room temperature to prevent the interdiffusion process of polymer chains between the labeled layer and unlabeled layer. Because the adsorption process is typically very slow (several hours) for long polymer chains even at $T \gg T_{\mathrm{g}}{ }^{24}$ the resultant structure of the adsorbed layer formed in Ellison and Torkelson's ${ }^{22}$ work would be quite different from that found in our study, resulting in quite different substrate effects. Further studies are in progress to clarify this point.

\section{CONCLUSIONS}

In this article, we highlight our recent work on the effect of nanoscale confinement on the local viscosity distributions within single PS nanoconfined films in equilibrium. The technique used was marker GIXPCS with resonance-enhanced X-ray scattering. We found that (i) the Brownian motion of the Au nanoparticle markers adequately tracks the viscosity of the polymer matrix via the Stokes-Einstein relationship and (ii) the marker motion primarily probes the entangled dynamics of polymer chains in the regions of interest. With this novel technique, we explored the inherent viscoelastic properties at the air/polymer interface and polymer/substrate interface and their roles in determination of the local viscosity distributions within single PS nanoconfined films at temperatures much greater than the bulk glass transition temperature. Because $\mathrm{Au}$ is known to be inert and has been specifically approved by the US Food and Drug Administration for internal medical use, the marker GIXPCS technique would open up a new avenue to explore in situ and perturbation-free dynamics at specific surfaces and interfaces of biopolymer films/membranes. Needless to say, other inorganic nanoparticles can be used as markers, expanding the applications of marker GIXPCS for interdisciplinary materials research. In addition, the advent of an X-ray free-electron laser would further provide a unique opportunity for marker GIXPCS to identify nonequilibrium chemical and biological phenomena with unprecedented temporal resolution (femtosecond regime).

\section{ACKNOWLEDGEMENTS}

We thank Peter Gin, Chunhua Li, Jaseung Koo, Miriam Rafailovich, Dong Ryeol Lee, Suresh Narayanan, Laurence Lurio and Sunil Sinha for their contributions to the present marker XPCS results. Funding was provided by NSF (grant no. CMMI-084626). Use of the Advanced Photon Source was supported by the US DOE under contract no. DE-AC02-06CH11357.
1 Keddie, J. L., Jones, R. A. L. \& Cory, R. A. Interface and surface effects on the glasstransition temperature in thin polymer-films. Faraday Discuss. 98, 219-230 (1994).

2 Keddie, J. L., Jones, R. A. L. \& Cory, R. A. Size-dependent depression of the glasstransition temperature in polymer-films. Europhys. Lett. 27, 59-64 (1994).

$3 \mathrm{Kim}$, J. H., Jang, J. \& Zin, W. C. Estimation of the thickness dependence of the glass transition temperature in various thin polymer films. Langmuir 16, 4064-4067 (2000).

4 Kawana, S. \& Jones, R. A. L. Character of the glass transition in thin supported polymer films. Phys. Rev. E. 63, 021501 (2001).

5 Xie, F. C., Zhang, H. F., Lee, F. K., Du, B. Y., Tsui, O. K. C., Yokoe, Y., Tanaka, K., Takahara, A., Kajiyama, T. \& He, T. B. Effect of low surface energy chain ends on the glass transition temperature of polymer thin films. Macromolecules 35, 1491-1492 (2002).

6 Fakhraai, Z., Valadkhan, S. \& Forrest, J. A. Qualitative discrepancy between different measures of dynamics in thin polymer films. Eur. Phys. J. E. 18, 143-148 (2005).

7 Fakhraai, Z. \& Forrest, J. A. Probing slow dynamics in supported thin polymer films. Phys. Rev. Lett. 95, 025701 (2005).

8 Kajiyama, T., Tanaka, K. \& Takahara, A. Depth dependence of the surface glasstransition temperature of a poly(styrene-block-methyl methacrylate) diblock copolymer film on the basis of temperature-dependent x-ray photoelectron-spectroscopy. Macromolecules 28, 3482-3484 (1995).

9 Mattsson, J., Forrest, J. A. \& Borjesson, L. Quantifying glass transition behavior in ultrathin free-standing polymer films. Phys. Rev. E. 62, 5187-5200 (2000).

10 Forrest, J. A., DalnokiVeress, K. \& Dutcher, J. R. Interface and chain confinement effects on the glass transition temperature of thin polymer films. Phys. Rev. E. 56, 5705-5716 (1997).

11 Forrest, J. A., DalnokiVeress, K., Stevens, J. R. \& Dutcher, J. R. Effect of free surfaces on the glass transition temperature of thin polymer films. Phys. Rev. Lett. 77, 2002-2005 (1996).

12 Teichroeb, J. H. \& Forrest, J. A. Direct imaging of nanoparticle embedding to probe viscoelasticity of polymer surfaces. Phys. Rev. Lett. 91, 016104 (2003).

13 Qi, D., Ilton, M. \& Forrest, J. A. Measuring surface and bulk relaxation in glassy polymers. Eur. Phys. J. E. 34, 1-7 (2011)

14 Ilton, M., Qi, D. \& Forrest, J. A. Using nanoparticle embedding to probe surface rheology and the length scale of surface mobility in glassy polymers. Macromolecules 42, 6851-6854 (2009).

15 Qi, D., Fakhraai, Z. \& Forrest, J. A. Substrate and chain size dependence of near surface dynamics of glassy polymers. Phys. Rev. Lett. 101, 096101 (2008).

16 Fakhraai, Z. \& Forrest, J. A. Measuring the surface dynamics of glassy polymers. Science 319, 600-604 (2008).

17 Sharp, J. S., Teichroeb, J. H. \& Forrest, J. A. The properties of free polymer surfaces and their influence on the glass transition temperature of thin polystyrene films. Eur. Phys. J. E. 15, 473-487 (2004).

18 Tanaka, K., Takahara, A. \& Kajiyama, T. Effect of polydispersity on surface molecular motion of polystyrene films. Macromolecules 30, 6626-6632 (1997).

19 DeMaggio, G. B., Frieze, W. E., Gidley, D. W., Zhu, M., Hristov, H. A. \& Yee, A. F. Interface and surface effects on the glass transition in thin polystyrene films. Phys. Rev. Lett. 78, 1524-1527 (1997).

20 Roth, C. B., McNerny, K. L. Jager, W. F. \& Torkelson, J. M. Eliminating the enhanced mobility at the free surface of polystyrene: fluorescence studies of the glass transition temperature in thin bilayer films of immiscible polymers. Macromolecules 40, 2568-2574 (2007).

21 Priestley, R. D., Ellison, C. J., Broadbelt, L. J. \& Torkelson, J. M. Structural relaxation of polymer glasses at surfaces, interfaces and in between. Science 309, 456-459 (2005).

22 Ellison, C. J. \& Torkelson, J. M. The distribution of glass-transition temperatures in nanoscopically confined glass formers. Nat. Mater. 2, 695-700 (2003).

23 Rotella, C., Wubbenhorst, M. \& Napolitano, S. Probing interfacial mobility profiles via the impact of nanoscopic confinement on the strength of the dynamic glass transition. Soft. Matter 7, 5260-5266 (2011).

24 Napolitano, S. \& Wubbenhorst, M. The lifetime of the deviations from bulk behaviour in polymers confined at the nanoscale. Nat. Commun. 2 (2011).

25 Rotella, C., Napolitano, S., De Cremer, L., Koeckelberghs, G. \& Wubbenhorst, M. Distribution of segmental mobility in ultrathin polymer films. Macromolecules 43, 8686-8691 (2010).

26 Napolitano, S. \& Wubbenhorst, M. Structural relaxation and dynamic fragility of freely standing polymer films. Polymer (Guildf) 51, 5309-5312 (2010).

27 Rotella, C., Napolitano, S. \& Wuebbenhorst, M. Segmental mobility and glass transition temperature of freely suspended ultrathin polymer membranes. Macromolecules 42, 1415-1417 (2009).

28 Peter, S., Napolitano, S., Meyer, H., Wubbenhorst, M. \& Baschnagel, J. Modeling dielectric relaxation in polymer glass simulations: dynamics in the bulk and in supported polymer films. Macromolecules 41, 7729-7743 (2008).

29 Napolitano, S., Lupascu, V. \& Wubbenhorst, M. Temperature dependence of the deviations from bulk behavior in ultrathin polymer films. Macromolecules 41, 1061-1063 (2008).

30 Napolitano, S. \& Wubbenhorst, M. Effect of a reduced mobility layer on the interplay between molecular relaxations and diffusion-limited crystallization rate in ultrathin polymer films. J. Phys. Chem. B. 111, 5775-5780 (2007).

31 Napolitano, S., Prevosto, D., Lucchesi, M., Pingue, P., D’Acunto, M. \& Rolla, P. Influence of a reduced mobility layer on the structural relaxation dynamics of 
aluminum capped ultrathin films of poly(ethylene terephthalate). Langmuir 23, 21032109 (2007)

32 Kessairi, K., Napolitano, S., Capaccioli, S., Rolla, P. \& Wubbenhorst, M. Molecular dynamics of atactic poly(propylene) investigated by broadband dielectric spectroscopy. Macromolecules 40, 1786-1788 (2007).

33 Wallace, W. E., Vanzanten, J. H. \& Wu, W. L. Influence of an impenetrable interface on a polymer glass-transition temperature. Phys. Rev. E. 52, R3329-R3332 (1995).

34 van Zanten, J. H., Wallace, W. E. \& Wu, W. L. Effect of strongly favorable substrate interactions on the thermal properties of ultrathin polymer Films. Phys. Rev. E. 53, R2053-R2056 (1996).

35 Fryer, D. S., Peters, R. D., Kim, E. J., Tomaszewski, J. E., de Pablo, J. J., Nealey, P. F., White, C. C. \& Wu, W. L. Dependence of the glass transition temperature of polymer films on interfacial energy and thickness. Macromolecules 34, 5627-5634 (2001).

36 Kim, H., Ruhm, A., Lurio, L. B., Basu, J. K., Lal, J., Lumma, D., Mochrie, S. G. J. \& Sinha, S. K. Surface dynamics of polymer films. Phys. Rev. Lett. 90, 068302 (2003).

$37 \mathrm{Kim}, \mathrm{H}$., Ruhm, A., Lurio, L. B., Basu, J. K., Lal, J., Mochrie, S. G. J. \& Sinha, S. K. $\mathrm{X}$-ray photon correlation spectroscopy on polymer films with molecular weight dependence. Physica. B. 336, 211-215 (2003).

38 Li, C. H., Koga, T., Jiang, J., Sharma, S., Narayanan, S., Lurio, L. B., Hu, Y., Jiao, X., Sinha, S. K., Billet, S., Sosnowik, D., Kim, H., Sokolov, J. C. \& Rafailovich, M. H. Viscosity measurements of very thin polymer films. Macromolecules 38, 5144-5151 (2005).

39 Koga, T., Li, C., Endoh, M. K., Koo, J., Rafailovich, M., Narayanan, S., Lee, D. R., Lurio, L. B. \& Sinha, S. K. Reduced viscosity of the free surface in entangled polymer melt films. Phys. Rev. Lett. 104, 225901-1-4 (2010).

40 Koga, T., Jiang, N., Gin, P., Endoh, M. K., Narayanan, S., Lurio, L. B. \& Sinha, S. K. Impact of an irreversibly adsorbed layer on local viscosity of nanoconfined polymer melts. Phys. Rev. Lett. 107, 066101-1-5 (2011).

41 Chu, B. Dynamic Light Scattering. 2nd Ed. (Academic Press, New York, 1991).

42 Leheny, R. L. Xpcs: nanoscale motion and rheology. Curr. Opin. Colloid. In. 17, 3-12 (2012).

43 Sutton, M. A. Review of x-ray intensity fluctuation spectroscopy. Cr. Phys. 9, 657-667 (2008).

44 Livet, F. Diffraction with a coherent x-ray beam: dynamics and imaging. Acta. Crystallogr. A. 63, 87-107 (2007).

45 Mochrie, S. G. J., Lurio, L. B., Ruhm, A., Lumma, D., Borthwick, M., Falus, P., Kim, H. J., Basu, J. K., Lal, J. \& Sinha, S. K. X-ray photon correlation spectroscopy studies of colloidal diffusion and the capillary modes of polymer films. Physica. B. 336, 173-180 (2003).

46 Kim, H., Ruhm, A., Lurio, L. B., Basu, J. K., Lal, J., Mochrie, S. G. J. \& Sinha, S. K. Synchrotron radiation studies of the dynamics of polymer films. J. Phys-Condens. Mat 16, S3491-S3497 (2004)

47 Jiang, Z., Kim, H., Jiao, X., Lee, H., Lee, Y. J., Byun, Y., Song, S., Eom, D., Li, C., Rafailovich, M. H., Lurio, L. B. \& Sinha, S. K. Evidence for viscoelastic effects in surface capillary waves of molten polymer films. Phys. Rev. Lett. 98, 227801-1-4 (2007).

48 Kim, H., Jiang, Z., Lee, H., Lee, Y. J., Jiao, X. S., Li, C. H., Lurio, L., Rafailovich, M. \& Sinha, S. K. Hydrodynamic surface fluctuations of polymer films by coherent x-ray scattering. Thin Solid Films 515, 5536-5540 (2007).

49 Jiang, Z., Mukhopadhyay, M. K., Song, S., Narayanan, S., Lurio, L. B., Kim, H. \& Sinha, S. K. Entanglement effects in capillary waves on liquid polymer films. Phys. Rev. Lett. 101, 246104-1-4 (2008).
50 Dierker, S. B., Pindak, R., Fleming, R. M., Robinson, I. K. \& Berman, L. X-ray photoncorrelation spectroscopy study of brownian-motion of gold colloids in glycerol. Phys. Rev. Lett. 75, 449-452 (1995).

51 Yee, C. K., Jordan, R., Ulman, A., White, H., King, A., Rafailovich, M. \& Sokolov, J. Novel one-phase synthesis of thiol-fuctionalized gold, palladium, and iridium nanoparticles using superhydride. Langmuir 15, 3486-3491 (1999).

52 Jiang, Z. X-ray scattering studies of structure and dynamics of surfaces and interfaces of polymeric liquids. Ph.D. dissertation, University of California, San Diego, b5455306 (2007).

53 Wang, J., Bedzyk, M. J. \& Caffrey, M. Resonance-enhanced $x$-rays in thinfilms - a structure probe for membranes and surface-layers. Science $258,775-778$ (1992)

54 Guo, H. Y., Bourret, G., Corbierre, M. K., Rucareanu, S., Lennox, R. B., Laaziri, K., Piche, L., Sutton, M., Harden, J. L. \& Leheny, R. L. Nanoparticle motion within glassy polymer melts. Phys. Rev. Lett. 102, 075702 (2009).

55 Narayanan, S., Lee, D. R., Hagman, A., Li, X. F. \& Wang, J. Particle dynamics in polymer-metal nanocomposite thin films on nanometer-length scales. Phys. Rev. Lett. 98, 185506-1-4 (2007).

56 Bodiguel, H. \& Fretigny, C. Reduced viscosity in thin polymer films. Phys. Rev. Lett. 97, 266105-1-4 (2006).

57 Bodiguel, H. \& Fretigny, C. Viscoelastic properties of ultrathin polystyrene films. Macromolecules 40, 7291-7298 (2007).

58 Ferry, J. D. Viscoelastic Properties of Polymers (Wiley, 1980).

59 Yang, Z. H., Clough, A., Lam, C. H. \& Tsui, O. K. C. Glass transition dynamics and surface mobility of entangled polystyrene films at equilibrium. Macromolecules 44, 8294-8300 (2011).

60 Rathfon, J. M., Cohn, R. W., Crosby, A. J., Rothstein, J. P. \& Tew, G. N. Confinement effects on chain entanglement in free-standing polystyrene ultrathin films. Macromolecules 44, 5436-5442 (2011).

61 Tsui, O. K. C. \& Zhang, H. F. Effects of chain ends and chain entanglement on the glass transition temperature of polymer thin films. Macromolecules 34, 9139-9142 (2001).

62 Mayes, A. M. Glass transition of amorphous polymer surfaces. Macromolecules 27, 3114-3115 (1994).

63 Brown, H. R. \& Russell, T. P. Entanglements at polymer surfaces and interfaces. Macromolecules 29, 798-800 (1996).

64 Si, L., Massa, M. V., Dalnoki-Veress, K., Brown, H. R. \& Jones, R. A. L. Chain entanglement in thin freestanding polymer films. Phys. Rev. Lett. 94, 127801-1-4 (2005).

65 Fetters, L. J., Lohse, D. J., Milner, S. T. \& Graessley, W. W. Packing length influence in linear polymer melts on the entanglement, critical, and reptation molecular weights. Macromolecules 32, 6847-6851 (1999).

66 Graessley, W. W. Some phenomenological consequences of the doi-edwards theory of viscoelasticity. J. Polym. Sci. Part B: Polym. Phys. 18, 27 (1980).

67 Fujii, Y., Yang, Z. H., Leach, J., Atarashi, H., Tanaka, K. \& Tsui, O. K. C. Affinity of polystyrene films to hydrogen-passivated silicon and its relevance to the t-g of the films. Macromolecules 42, 7418-7422 (2009).

68 Napolitano, S. \& Wubbenhorst, M. Dielectric signature of a dead layer in ultrathin films of a nonpolar polymer. J. Phys. Chem. B 111, 9197-9199 (2007).

69 Bruinsma, R. Slow Spreading of polymer melts. Macromolecules 23, 276-280 (1990)

70 Baljon, A., Billen, J. \& Khare, R. Phys. Rev. Lett. 93, 255701 (2004). 


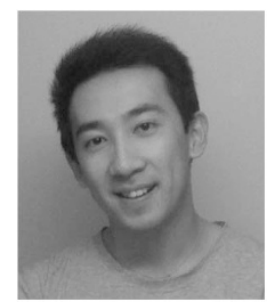

Naisheng Jiang is currently a $\mathrm{PhD}$ student of Materials Science and Engineering Department at Stony Brook University under the supervision of Professor Koga. He was born in Jiangsu, China in 6 March 1987. He received his Bachelor of Engineering Degree (2009) in Polymer Materials and Engineering at Sichuan University, China. His current research focuses on nanoconfinement effects of polymeric materials on such as melting/crystallization, dewetting and viscoelastic property. A variety of in-situ and real-time X-ray/ neutron scattering techniques in conjunction with atomic force microscopy have been employed in his research.

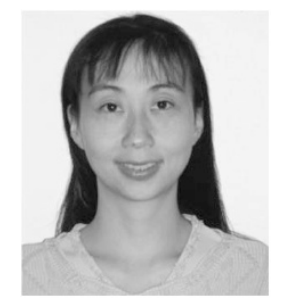

Maya K Endoh is a currently senior postdoctoral researcher at Stony Brook University. She received her Bachelor degree in polymer science (1995), Master degree in polymer science (1997) and her PhD degree in polymer physics (2005) from Kyoto University, Japan. She received the Restart Postdoctoral Fellowship (RPD) supported by Japan Society for the Promotion of Science from 2006 to 2008. Her research topics are phase transition/separation and dynamics of various polymers (including bulks, solutions and thin films) under non-liner, non-equilibrium state in a flow and a static field. She specializes in the structure characterization via X-ray/laser light scattering techniques. Her current interests extend to environmentally green polymer processing using supercritical fluids and methane hydrate as a future energy resource.

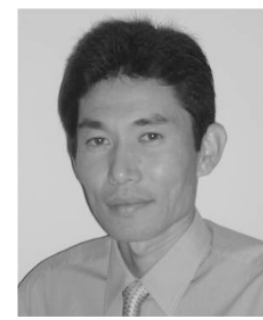

Tadanori (Tad) Koga received his master degree in Physics (1993) and PhD degree in Physics from Kyushu University, Japan (1998). During his PhD course, he joined the Hashimoto Polymer Phasing Project (ERATO, JST, Japan) as a research member, allowing him to establish a new foundation in polymer physics. He completed his postdoctoral work at Departments of Chemistry and Materials Science and Engineering at State University of New York at Stony Brook (currently known as Stony Brook University), before joining the Chemical and Molecular Engineering program at Stony Brook University as an Assistant Professor in 2006. He is currently an Associate Professor. He has extensive research experience in the areas of structure, property and functionality relationships in polymers along with the development of new 'green' process environments for polymer surfaces/interfaces. He is a recipient of the National Science Foundation (NSF) CAREER award, the NSF's most prestigious award in support of junior faculty. He has published more than 60 peer-reviewed scientific articles and 2 issues and 2 pending patents. He is currently the spokesperson of the X27C Beamline in National Synchrotron Light Source (NSLS), the first synchrotron facility in the United States dedicated to polymer research. 